Document downloaded from:

http://hdl.handle.net/10251/166352

This paper must be cited as:

Lizama, C.; Murillo Arcila, M.; Peris Manguillot, A. (2020). Nonlocal operators are chaotic. Chaos An Interdisciplinary Journal of Nonlinear Science. 30(10):1-8. https://doi.org/10.1063/5.0018408

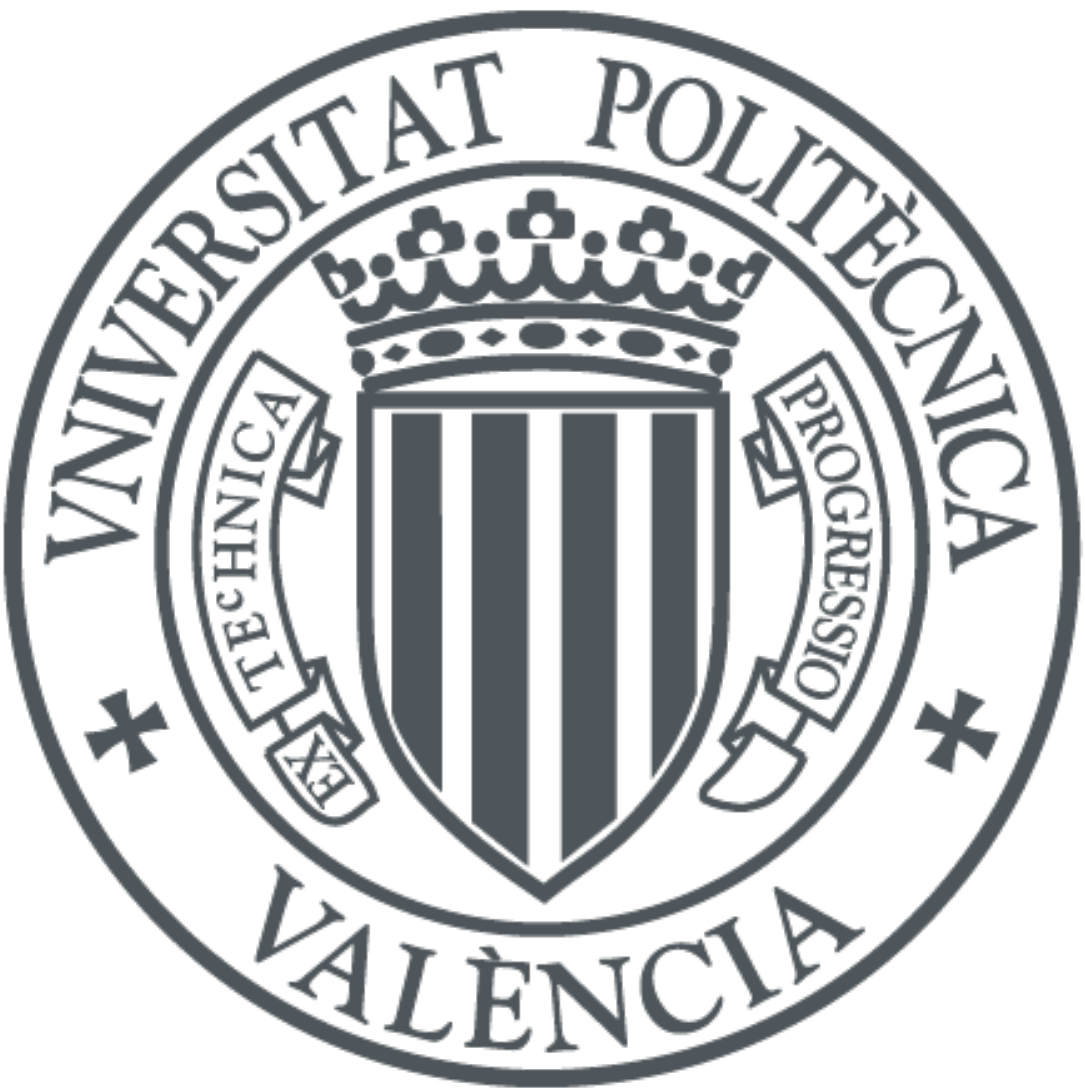

The final publication is available at https://doi.org/10.1063/5.0018408

Copyright American Institute of Physics

Additional Information 


\title{
NONLOCAL OPERATORS ARE CHAOTIC
}

\author{
CARLOS LIZAMA \\ DEPARTAMENTO DE MATEMÁtiCA Y CIENCIA DE LA COMPUTACIÓN, FACULTAD DE CIENCIAS, UNIVERSIDAD DE \\ SANTIAGo DE CHILE, CASILla 307, CORREO 2, SANTIAGO, CHILE. \\ EMAIL: CARLOS.LIZAMA@USACH.CL \\ MARINA MURILLO-ARCILA AND ALFRED PERIS \\ INSTITUTO UNIVERSITARIO DE MATEMÁTICA PURA Y APLICADA, UNIVERSITAT POLITÈCNICA DE VAL̀ENCIA, E-46022, \\ VALÈNCIA, SPAIN. \\ EMAILS: MAMUAR1@UPV.ES; APERIS@MAT.UPV.ES
}

\begin{abstract}
We characterize for the first time the chaotic behavior of nonlocal operators that come from a broad class of time stepping schemes of approximation for fractional differential operators. For that purpose, we use criteria for chaos of Toeplitz operators in Lebesgue spaces of sequences. Surprisingly, this characterization is proved to be - in some cases - dependent of the fractional order of the operator and the step size of the scheme.
\end{abstract}

The study of discrete nonlocal operators, having fractional order operators as a prototype, is a fashionable area of study nowadays. As it has been recently demonstrated, systems with this property can be used to develop data encryption algorithms. The basis for such property is the chaotic behavior of the systems under consideration. With this motivation, we investigate necessary and sufficient conditions for chaos, uniquely based on the available data of nonlocal operators arising in numerical analysis, namely, the fractional order and the step size. We have success in our investigation and having our new findings somewhat surprising: We find that for the dual of the operator that defines the second order backward fractional difference scheme chaos is dependent on the step size but not on the fractional order and, by contrast, in case of the fractional Crank-Nicholson scheme chaos is dependent on the step size and the fractional order. Consequently, our new findings have ultimate importante in the development of algorithms based on the mentioned schemes of approximation, among others, which are also analyzed in the present research.

\section{INTRODUCTION}

In the last two decades, due to the introduction of fractional (or non-local) operators in the field of mathematical modeling of problems that arise in various areas of knowledge, many researchers have experienced a great interest in deeply understanding the behavior of these operators from different perspectives [15, 16, 17, 19, 22. One of the main efforts has been made to connect these operators with the modeling of partial differential equations, as a new method to understand the processes that are closely related to having "memory" in their nature. On the other hand, it is well known that complex partial differential equations modeling needs an in-depth analysis of linear evolution systems.

Linear evolution equations described by non-local operators have been studied intensively in the last decade [6, 20, 21, 26]. One of the most challenging problems in this context is trying to find the most appropriate discretization of the fractional operator in each case. This choice is determined by the nature of the evolution equation and the geometry of the model. These studies have led researchers to adapt various classical numerical methods to the context of non-local operators in order to discretize them in time and/or space.

2010 Mathematics Subject Classification. 47A16; 47B35; 34A08; 65M60.

Key words and phrases. Nonlocal operators; Toeplitz operators; Chaos; time stepping schemes; transference principle. 
Recently, a very interesting approach has been proposed by Jin, Li and Zhou in [25] to deal in a unified way with a large number of time discrete non-local operators that arise in the numerical analysis of fractional order differential operators. This method can synthesize by means of finite convolution several numerical methods, such as Euler's backward and implicit schemes and the Crank-Nicholson scheme among others.

Although the numerical treatment has been carried out in [25], a qualitative analysis of these non-local discrete operators has not been performed yet. This is a difficult challenge because, due to the nature of the subject, it needs the interaction between different areas of mathematics such as: functional, complex and numerical analysis and operator theory among others.

From an analytical perspective, the study of the chaotic behavior is an important first step that began recently to be studied by Edelman [13, 12, 14] and Wu et.al. [34, 35]. The reason is that, by their very nature, non-local operators seem naturally linked to a complex evolution. On the other hand, due to the high sensitivity of chaotic systems to parameters and initial conditions, chaos based algorithms are developed and studied as the core of encryption algorithms [32, 33. For instance, many substitution encryption algorithms have been introduced based on discrete chaotic maps such as the conventional logistic and tent maps. This motivates the following questions: Is there any kind of chaotic behavior associated with the time approximation schemes for non-local (fractional) differential operators? If the answer is positive, is there any dependence on the chaos with respect to the fractional order and / or the size of the scheme step?

In this article we answer in the positive the aforementioned questions by studying a broad class of nonlocal operators that include those given in the form of a finite convolution operator, as motivated by the reference [25]. To achieve this goal, we extend a result due to Baranov and Lishanskii [8, which provides a first criterion of chaos for Toeplitz operators to deal with symbols associated to numerical methods that have a singular behavior at zero, but no more than a simple pole. The dynamics of such operators have been also considered in [10, 30, 31. We also obtain a new spectral characterization of the chaotic behavior for the dual convolution operator defined in Lebesgue sequence spaces.

As a consequence, we are able to show the chaotic behavior of the classical fractional difference operator $\nabla_{a}^{\alpha}$ defined by Atici and Eloe [2, 3, 4, 5] and Gray and Zhang [23], but our more surprising finding is the chaotic dependence of some non-local operators, either in the fractional order or in the size of the scheme's time step. For example, in the case of the fractional explicit Euler scheme (for fractional order $0<\alpha \leq 1$ ), the subjacent non-local operator defines a chaotic Toeplitz operator on $\ell^{2}\left(\mathbb{N}_{0}\right)$. In the situation of the fractional implicit Euler scheme, we find that the fractional order Weil difference operator is chaotic on $\ell^{2}\left(\mathbb{N}_{0}\right)$ if and only if the step size $\tau$ of the scheme belongs to the interval $0<\tau<2$. In contrast, given $0<\alpha<1$, the non-local (and dual) operator associated to the fractional Crank-Nicholson scheme with step size $\tau$, is chaotic on $\ell^{2}\left(\mathbb{N}_{0}\right)$ if and only if $0<\tau<\frac{2}{(1-\alpha)^{1 / \alpha}}$.

The paper is organized as follows: Section 2 is devoted to recall some preliminaries related to chaotic operators (in the sense of Devaney) and a useful criterion for hypercyclicity of Toeplitz operators. Section 3 deals with the proof of a new and important transference principle that allows to transfer results from the particular case of the operator $\Delta^{\alpha} \equiv \nabla_{0}^{\alpha}$ to the general case by means of conjugation by translation. This new technique has been recently proved to be very useful in the treatment of qualitative properties for fractional difference operators [22]. Section 4 contains an extension of existing criteria for chaos of Toeplitz operators, that we will later need to prove the chaotic behavior of nonlocal operators. Section 5 characterizes, in Lebesgue spaces of sequences, the chaotic behavior of nonlocal fractional discrete operators coming from approximation schemes in terms of the scheme step size and fractional order.

\section{Preliminaries}

We recall that an operator $T$ on a topological vector space $X$ is called hypercyclic if there is a vector $x$ in $X$ such that its orbit $\operatorname{Orb}(x, T)=\left\{x, T x, T^{2} x, \ldots\right\}$ is dense in $X$.

According to [11 an operator $T$ on a topological vector space $X$ is Devaney chaotic if it is hypercyclic, the set of periodic points $\operatorname{Per}(T)$ is dense in $X$, and it is sensitive (i.e., there exists $\epsilon>0$ 
such that for each $x \in X$ and each $\delta>0$ there are $y \in X$ and $n \in \mathbb{N}$ with $d(x, y)<\delta$ and $\left.d\left(T^{n}(x), T^{n}(y)\right)>\epsilon\right)$. It is well-known that if we have the first two conditions for any continuous map $T$ on a metric space $X$ without isolated points then sensitivity is granted [7. Given two operators $T$ and $S$ defined on topological vector spaces $X$ and $Y$, respectively, we say $T$ is quasi-conjugate to $S$ if there exists a continuous map $\Phi: Y \rightarrow X$ with dense range such that $T \circ \Phi=\Phi \circ S$. The recent books [9] and 24] contain the theory and most of the recent advances on hypercyclicity and linear dynamics.

In what follows we denote by $\overline{\mathbb{D}}$ the closed unit disk and put $\widehat{\mathbb{D}}=\mathbb{C} \backslash \overline{\mathbb{D}}$. We recall that a Toeplitz operator $T_{\Phi}: \mathcal{H}^{2}(\mathbb{D}) \rightarrow \mathcal{H}^{2}(\mathbb{D})$ with symbol $\Phi \in L^{\infty}(\mathbb{T})$ is defined by $T_{\Phi}(f)=P\left(M_{\Phi}(f)\right), f \in \mathcal{H}^{2}(\mathbb{D})$, where $M_{\Phi}$ is the multiplication operator by $\Phi$ and $P: L^{2}(\mathbb{T}) \rightarrow \mathcal{H}^{2}(\mathbb{D})$ is the Riesz projection. If we write

$$
\Phi(z)=\sum_{n \in \mathbb{Z}} a_{n} z^{n} \in L^{\infty}(\mathbb{T})
$$

given $f(z)=\sum_{n=0}^{\infty} b_{n} z^{n} \in \mathcal{H}^{2}(\mathbb{D})$, we can write $\left(T_{\Phi} f\right)(z)=\sum_{n=0}^{\infty} c_{n} z^{n} \in \mathcal{H}^{2}(\mathbb{D})$, where the sequence $c=\left(c_{n}\right)_{n}$ is obtained as the convolution of $a=\left(a_{n}\right)_{n}$ with $b=\left(b_{n}\right)_{n}$, i.e. $c=a * b$, defined as

$$
c_{n}=(a * b)_{n}=\sum_{j=-\infty}^{n} a_{j} b_{n-j}, \quad n \in \mathbb{N}_{0} .
$$

That is, the Toeplitz operator is then considered as an (infinite matrix) operator $T_{\Phi}: \ell^{2}\left(\mathbb{N}_{0}\right) \rightarrow \ell^{2}\left(\mathbb{N}_{0}\right)$ as the above convolution. In case that $\Phi(z)=\sum_{n \in \mathbb{Z}} a_{n} z^{n}$ is so that $a=\left(a_{n}\right)_{n} \in \ell^{1}(\mathbb{Z})$, then $T_{\Phi}$ is a well defined bounded operator on $\ell^{2}\left(\mathbb{N}_{0}\right)$.

Let $\Phi(\mathbb{D}, N):=\{\omega \in \mathbb{C}:$ the equation $\Phi(z)=\omega$ has $N$ solutions in $\mathbb{D}\}$. In [8] the authors provide sufficient and necessary conditions that ensure hypercyclicity of the Toeplitz operator $T_{\Phi}$ in the case $\Phi(z)=p\left(\frac{1}{z}\right)+\varphi(z)$ where $p$ is a polynomial and $\varphi \in H^{\infty}$. They pay special attention to the case $p(z)=\gamma z$ and prove the following interesting result.

Theorem 2.1 ([] $]$ ). Let $\gamma \in \mathbb{C}$, let $\varphi \in H^{\infty}$ and let $\Phi(z)=\frac{\gamma}{z}+\varphi(z)$.

(a) If $T_{\Phi}$ is hypercyclic then

(i) the function $\Phi$ is univalent in $\mathbb{D} \backslash\{0\}$;

(ii) $\mathbb{D} \cap(\mathbb{C} \backslash \Phi(\mathbb{D})) \neq \emptyset$ and $\widehat{\mathbb{D}} \cap(\mathbb{C} \backslash \Phi(\mathbb{D})) \neq \emptyset$.

(b) If $\varphi \in A(\overline{\mathbb{D}})$ and

(i) the function $\Phi$ is univalent in $\overline{\mathbb{D}} \backslash\{0\}$;

(ii) $\mathbb{D} \cap(\mathbb{C} \backslash \Phi(\mathbb{D})) \neq \emptyset$ and $\widehat{\mathbb{D}} \cap(\mathbb{C} \backslash \Phi(\mathbb{D})) \neq \emptyset$. then $T_{\Phi}$ is hypercyclic.

Concerning the spectrum of the hypercyclic operator, they show the next proposition.

Proposition $2.2([8])$. Assume that $\Phi$ is $N$-valent in $\mathbb{D}$. Then

$$
\sigma\left(T_{\Phi}\right)=\mathbb{C} \backslash \Phi(\mathbb{D}, N), \quad \mathbb{C} \backslash \overline{\Phi(\mathbb{D})} \subset \sigma\left(T_{\Phi}\right) .
$$

If $\lambda \in \mathbb{C} \backslash \overline{\Phi(\mathbb{D})}$ then the corresponding eigenspace has dimension $N$ and the eigenvectors are given by

$$
f_{\lambda}(z)=\frac{q(z)}{z^{N} \Phi(z)-\lambda z^{N}}
$$

where $q$ is an arbitrary polynomial of degree at most $N-1$.

In particular, for univalent $\Phi$, we get that

$$
f_{\lambda}(z)=\frac{1}{z \Phi(z)-\lambda z}
$$

is a $\lambda$-eigenvector of $T_{\Phi}$ for any $\lambda \in \mathbb{C} \backslash \overline{\Phi(\mathbb{D})}$.

Let $B$ be denote the backward shift operator on $\ell^{2}\left(\mathbb{N}_{0}\right)$, and observe that $\|B\|=1$. Let $a \in \ell^{1}\left(\mathbb{N}_{0}\right)$ be a complex valued sequence. Hence, if $\varphi(z)=\sum_{n=0}^{\infty} a_{n} z^{n}$ is holomorphic on some neighborhood of 
$\overline{\mathbb{D}}$ then

$$
\varphi(B)=\sum_{n=0}^{\infty} a_{n} B^{n}
$$

defines a bounded operator on $\ell^{2}\left(\mathbb{N}_{0}\right)$. Denote by $\mathbb{T}$ the unit circle. The following characterization will be very useful in our analysis.

Theorem 2.3. 24, Theorem 4.43] Let $X$ be one of the complex sequence spaces $\ell^{2}\left(\mathbb{N}_{0}\right)$. Furthermore, let $\varphi$ be a nonconstant holomorphic function on a neighborhood of $\overline{\mathbb{D}}$. Then the following assertions are equivalent.
(i) $\varphi(B)$ is chaotic;

(ii) $\varphi(\mathbb{D}) \cap \mathbb{T} \neq \emptyset$.

For further use, we also recall a useful criterion that ensures univalence for meromorphic functions [18.

Theorem 2.4. Let $M_{n}$ denote the class of functions of the form $f(z)=\frac{1}{z}+\sum_{n=0}^{\infty} a_{n} z^{n}$ which are regular in $0<|z|<1$ and satisfy

$$
\Re\left(\frac{D^{n+1} f(z)}{D^{n} f(z)}-2\right)<-\frac{n}{n+1}, \text { for }|z|<1
$$

where $D^{n} f(z)=\frac{1}{z}\left(z^{n+1} \frac{f(z)}{n !}\right)^{(n)}, n \in \mathbb{N}_{0}$. Then $M_{n+1} \subset M_{n}$ for all $n \in \mathbb{N}_{0}$ and all functions in $M_{n}$ are univalent.

\section{A transference PRINCIPLE BETWEEN NONLOCAL OPERATORS}

For a real number $a$, we denote $\mathbb{N}_{a}:=\{a, a+1, a+2, \ldots\}$. and we write $\mathbb{N}_{1} \equiv \mathbb{N}$. We denote by $s\left(\mathbb{N}_{a}\right)$ the vectorial space consisting of all complex-valued sequences $f: \mathbb{N}_{a} \rightarrow \mathbb{C}$. We recall that the forward Euler operator $\Delta_{a}: s\left(\mathbb{N}_{a}\right) \rightarrow s\left(\mathbb{N}_{a}\right)$ is defined by

$$
\Delta_{a} f(t):=f(t+1)-f(t), \quad t \in \mathbb{N}_{a}
$$

For $m \in \mathbb{N}_{2}$, we define recursively $\Delta_{a}^{m}: s\left(\mathbb{N}_{a}\right) \rightarrow s\left(\mathbb{N}_{a}\right)$ by $\Delta_{a}^{m}:=\Delta_{a}^{m-1} \circ \Delta_{a}$, and is called the $m$-th order forward difference operator. For instance, for any $f \in s\left(\mathbb{N}_{0}\right)$, we have

$$
\Delta^{m} f(n)=\sum_{j=0}^{m}\left(\begin{array}{c}
m \\
j
\end{array}\right)(-1)^{m-j} f(n+j), \quad n \in \mathbb{N}_{0} .
$$

where $\Delta \equiv \Delta_{0}$ and $\Delta_{a}^{0} \equiv I_{a}$, with $I_{a}: s\left(\mathbb{N}_{a}\right) \rightarrow s\left(\mathbb{N}_{a}\right)$ the identity operator. We define the translation (by $a \in \mathbb{R}$ ) operator $\tau_{a}: s\left(\mathbb{N}_{a}\right) \rightarrow s\left(\mathbb{N}_{0}\right)$ by $\tau_{a} g(n):=g(a+n), \quad n \in \mathbb{N}_{0}$. Note that $\tau_{a}^{-1}=\tau_{-a}$ and $\tau_{a+b}=\tau_{a} \circ \tau_{b}=\tau_{b} \circ \tau_{a}$. Moreover, $\Delta_{a}^{m} \circ \tau_{a}^{-1}=\tau_{a}^{-1} \circ \Delta^{m}$. In particular, we have

$$
\Delta_{a}^{m} f(a+n)=\sum_{j=0}^{m}\left(\begin{array}{c}
m \\
j
\end{array}\right)(-1)^{m-j} f(a+n+j), \quad n \in \mathbb{N}_{0} .
$$

For any $\alpha \in \mathbb{R} \backslash\{0\}$, we set

$$
k^{\alpha}(n)=\left\{\begin{array}{cc}
\frac{\alpha(\alpha+1) \ldots(\alpha+n-1)}{n !} & n \in \mathbb{N}_{0} \\
0 & \text { otherwise. }
\end{array}\right.
$$

In case $\alpha=0$ we define $k^{0}(n)=e_{0}(n)$ where $e_{i}(j)$ is the Kronecker delta. Note that if $\alpha \in \mathbb{R} \backslash$ $\{-1,-2, .$.$\} , we have k^{\alpha}(n)=\frac{\Gamma(\alpha+n)}{\Gamma(\alpha) \Gamma(n+1)}, n \in \mathbb{N}_{0}$ where $\Gamma$ is the Euler gamma function. From [28], or directly from the definition, we have the generation formula

$$
\sum_{j=0}^{\infty} k^{\beta}(j) z^{j}=\frac{1}{(1-z)^{\beta}}, \quad \beta \in \mathbb{R}, \quad|z|<1,
$$


see [36, p.42 formulae (1) and (8)]. The sequence $k^{\alpha}(n)$ will be very important in our analysis. Several properties are collected in the recent paper [22]. We recall the definition of $\alpha$-th fractional sum on the set $\mathbb{N}_{0}$.

Definition 3.1. For each $\alpha>0$ and $f \in s\left(\mathbb{N}_{0}\right)$, we define

$$
\Delta^{-\alpha} f(n):=\sum_{j=0}^{n} k^{\alpha}(n-j) f(j), \quad n \in \mathbb{N}_{0},
$$

Recall that the finite convolution $*$ of two sequences $f$ and $g$ is defined by

$$
(f * g)(n):=\sum_{j=0}^{n} f(n-j) g(j), \quad n \in \mathbb{N}_{0} .
$$

Therefore, by definition, we have $\Delta^{-\alpha} f(n)=\left(k^{\alpha} * f\right)(n), n \in \mathbb{N}_{0}$, where the convolution operator $*$ enjoys algebraic properties like commutativity and associativity, which will be very useful to simplify and better understand the proof of some results. The above concept comes from a more general definition, which is the most commonly used in the literature of fractional difference equations.

Definition 3.2. [5] Let $\alpha>0$. For any given positive real number $a$, the $\alpha$-th fractional sum of a function $f$ is

$$
\nabla_{a}^{-\alpha} f(t)=\frac{1}{\Gamma(\alpha)} \sum_{s=a}^{t}(t-s+1)^{\overline{\alpha-1}} f(s)
$$

where $t \in \mathbb{N}_{a}$ and $t^{\bar{\alpha}}:=\frac{\Gamma(t+\alpha)}{\Gamma(t)}$.

We next recall the definition of fractional difference operator, which was introduced in [27].

Definition 3.3. The fractional difference operator $\Delta^{\alpha}: s\left(\mathbb{N}_{0}\right) \rightarrow s\left(\mathbb{N}_{0}\right)$ of order $\alpha>0$ (in the sense of Riemann-Liouville) is defined by

$$
\Delta^{\alpha} f(n):=\Delta^{m} \circ \Delta^{-(m-\alpha)} f(n), \quad n \in \mathbb{N}_{0},
$$

where $m-1<\alpha<m, m:=\lceil\alpha\rceil$, the least integer that is greater than or equal to $\alpha$.

For instance, for $0<\alpha<1$ we obtain

$$
\Delta^{\alpha} f(n)=\sum_{j=0}^{n+1} k^{-\alpha}(j) f(n-j)=\sum_{j=0}^{n+1}(-1)^{j}\left(\begin{array}{c}
-\alpha \\
j
\end{array}\right) f(n-j),
$$

which corresponds to the Grünwald-Letnikov scheme of approximation (with step size $h=1$ ) for the one dimensional Caputo fractional derivative. Complementarily we recall the following definition.

Definition 3.4. The nabla fractional difference operator $\nabla^{\alpha}: s\left(\mathbb{N}_{a}\right) \rightarrow s\left(\mathbb{N}_{a}\right)$ of order $\alpha>0$ is defined by

$$
\nabla_{a}^{\alpha} f(t)=\Delta_{a}^{m} \circ \nabla_{a}^{-(m-\alpha)} f(t), \quad t \in \mathbb{N}_{a}
$$

where $m-1<\alpha<m, m=\lceil\alpha\rceil$.

In the next result we show an important relationship between the two above defined fractional difference operators, known as transference principle, because allows to transfer qualitative properties from one operator to another via translation. This kind of theorem was first proved in the article [22] where its usefulness in order to transfer geometric properties has been established.

Theorem 3.5. Let $\alpha>0$ and $a \in \mathbb{R}$ be given. Then we have

$$
\tau_{a} \circ \nabla_{a}^{\alpha}=\Delta^{\alpha} \circ \tau_{a} .
$$


Proof. By Definition 3.2 for $f \in s\left(\mathbb{N}_{a}\right)$ we have

$$
\begin{aligned}
\tau_{a} \circ \nabla_{a}^{-\alpha} f(n) & =\nabla_{a}^{-\alpha} f(n+a)=\frac{1}{\Gamma(\alpha)} \sum_{j=0}^{n}(n-j+1)^{\overline{\alpha-1}} f(a+j) \\
& =\sum_{j=0}^{n} \frac{\Gamma(\alpha+n-j)}{\Gamma(\alpha) \Gamma(n-j+1)} f(a+j)=\sum_{j=0}^{n} k^{\alpha}(n-j) f(a+j)=\Delta^{-\alpha} \circ \tau_{a} f(n),
\end{aligned}
$$

for all $n \in \mathbb{N}_{0}$. Let $f \in s\left(\mathbb{N}_{a}\right)$ be given. Then by Definition 3.4, identities (2), (3), (5) and (6), we obtain

$$
\begin{aligned}
\tau_{a} \circ \nabla_{a}^{\alpha} f(n) & =\tau_{a} \circ\left(\Delta_{a}^{m} \circ \nabla_{a}^{-(m-\alpha)}\right) f(n)=\left(\Delta_{a}^{m} \circ \nabla_{a}^{-(m-\alpha)}\right) f(n+a) \\
& =\sum_{j=0}^{m}\left(\begin{array}{c}
m \\
j
\end{array}\right)(-1)^{m-j} \nabla_{a}^{-(m-\alpha)} f(n+a+j) \\
& =\sum_{j=0}^{m}\left(\begin{array}{c}
m \\
j
\end{array}\right)(-1)^{m-j} \tau_{a} \circ \nabla_{a}^{-(m-\alpha)} f(n+j) \\
& =\sum_{j=0}^{m}\left(\begin{array}{c}
m \\
j
\end{array}\right)(-1)^{m-j} \Delta^{-(m-\alpha)} \circ \tau_{a} f(n+j) \\
& =\Delta^{m}\left(\Delta^{-(m-\alpha)} \circ \tau_{a} f\right)(n)=\Delta^{\alpha} \circ \tau_{a} f(n),
\end{aligned}
$$

for all $n \in \mathbb{N}_{0}$. This proves the theorem.

\section{Chaotic Toeplitz operators}

In this section we will show a new criterion in order to obtain (Devaney) chaos for the operators considered here. We recall that, given an operator $T: X \rightarrow X$ on a complex Banach space $X$, then a function $E: A \rightarrow X$ for certain $A \subset \mathbb{C}$ is an eigenvector field if $E(\lambda) \in \operatorname{ker}(\lambda I-T)$ for any $\lambda \in A$, and

$$
\operatorname{span}\{E(\lambda) ; \lambda \in A\} \text { is dense in } X
$$

Given a non-empty open set $U \subset \mathbb{C}$, and a map $G: U \rightarrow X$, we say that $G$ is weakly holomorphic on $U$ if $y \circ G: U \rightarrow \mathbb{C}$ is holomorphic for any $y \in X^{*}$.

The following result is essentially well-known. We will include its proof for the sake of completeness.

Theorem 4.1. Given an operator $T: X \rightarrow X$ on a complex Banach space $X$, if $U \subset \mathbb{C}$ is a connected nonempty open set that intersects $\mathbb{T}, G: U \rightarrow X$ is a weakly holomorphic map such that $G(\lambda) \in \operatorname{ker}(\lambda I-T)$ for any $\lambda \in U$, and

$$
\operatorname{span}\{G(\lambda) ; \lambda \in U\} \text { is dense in } X,
$$

then $T$ is Devaney chaotic.

Proof. By the classic Godefroy-Shapiro criterion for chaos (see, e.g., [24, Theorem 3.1]), we need to show that

$$
\begin{gathered}
Y_{1}:=\operatorname{span}\{x \in X ; T x=\lambda x \text { for some } \lambda \in \mathbb{C} \text { with }|\lambda|>1\} ; \\
Y_{2}:=\operatorname{span}\{x \in X ; T x=\lambda x \text { for some } \lambda \in \mathbb{C} \text { with }|\lambda|<1\} ; \\
Y_{3}:=\operatorname{span}\left\{x \in X ; T x=e^{\alpha \pi i} x \text { for some } \alpha \in \mathbb{Q}\right\} ;
\end{gathered}
$$

are dense in $X$. To do this, we consider $U_{1}:=U \cap(\mathbb{C} \backslash \overline{\mathbb{D}}), U_{2}:=U \cap \mathbb{D}$, and

$$
A:=U \cap\left\{e^{\alpha \pi i} ; \alpha \in \mathbb{Q}\right\} .
$$

It will suffice to show that, given $j \in\{1,2,3\}$, and for any $y \in X^{*}$, the equality $\langle x, y>=0$ for every $x \in Y_{j}$ implies $y=0$. Actually, since the holomorphic map $y \circ G$ annihilates on $U_{1}, U_{2}$ or $A$, which are sets with accumulating points in $U$, and $U$ is connected, then $y \circ G \equiv 0$. The assumptions imply that $y=0$, and we conclude the result. 
Now we are in conditions to recall the following theorem obtained in [8] that provides chaos for the kind of Toeplitz operators that we are interested. In the original paper, the authors only showed hypercyclicity for such operators but chaos can be easily reached.

Theorem 4.2. Let $\Phi(z)=\frac{\gamma}{z}+\varphi(z)$ with $\gamma \in \mathbb{C}$ and $\varphi \in A(\overline{\mathbb{D}})$ satisfying

(i) the function $\Phi$ is univalent in $\overline{\mathbb{D}} \backslash\{0\}$;

(ii) $\mathbb{D} \cap(\mathbb{C} \backslash \Phi(\mathbb{D})) \neq \emptyset$ and $\widehat{\mathbb{D}} \cap(\mathbb{C} \backslash \Phi(\mathbb{D})) \neq \emptyset$.

Then the Toeplitz operator $T_{\Phi}: \ell^{2}\left(\mathbb{N}_{0}\right) \rightarrow \ell^{2}\left(\mathbb{N}_{0}\right)$ is Devaney chaotic.

Proof. The fact that $T_{\Phi}$ is a well-defined hypercyclic operator on $\ell^{2}\left(\mathbb{N}_{0}\right)$ was proved by Baranov and Lishaskii [8, Theorem 1.1], and taking into account that they showed (see [8, Proposition 2.2]) that

$$
f_{\lambda}(z)=\frac{1}{z \Phi(z)-\lambda z} \in A(\mathbb{D})
$$

is a $\lambda$-eigenvector of $T_{\Phi}$ for any $\lambda \in \mathbb{C} \backslash \overline{\Phi(\mathbb{D})}$, we get that $G(\lambda):=f_{\lambda}$ is a weakly holomorphic map on an open $\operatorname{disc} U$ that intersects $\mathbb{T}$. Moreover, it satisfies the condition

$$
\operatorname{span}\{G(\lambda) ; \lambda \in U\} \text { is dense in } X \text {, }
$$

as was shown in the proof of statement 2 of Theorem 1.1 in 8 . Thus $T_{\Phi}$ is Devaney chaotic by Theorem 4.1

\section{Nonlocal operators are Chaotic}

Let $0<\alpha<1$. From the previous section, we recall the definition of the nonlocal operator $\Delta^{\alpha}$ which read as follows

$$
\Delta^{\alpha} u(n)=\Delta\left(k^{1-\alpha} * u\right)(n)=\sum_{j=0}^{n+1} k^{1-\alpha}(n+1-j) u(j)-\sum_{j=0}^{n} k^{1-\alpha}(n-j) u(j), \quad n \in \mathbb{N}_{0} .
$$

Our first important observation is that the representation of $\Delta^{\alpha}$ in the canonical basis $\left\{e_{l}(j)\right\}_{j, l \in \mathbb{N}_{0}}$ of $\ell^{2}\left(\mathbb{N}_{0}\right)$ is a Toeplitz matrix for $0<\alpha<1$. In fact, we have

$$
\Delta^{\alpha} e_{l}(n)= \begin{cases}-\alpha \frac{k^{1-\alpha}(n-l)}{n-l+1} & \text { if } n \geq l \\ 1 & \text { if } n=l-1 \\ 0 & \text { if } n<l-1\end{cases}
$$

Our next theorem shows that $\Delta^{\alpha}$ is a well-defined Toeplitz operator on $\ell^{2}\left(\mathbb{N}_{0}\right)$ and exhibits chaos for any $0<\alpha<1$.

Theorem 5.1. For any $0<\alpha<1$, the operator $\Delta^{\alpha}$ defines a chaotic Toeplitz operator on $\ell^{2}\left(\mathbb{N}_{0}\right)$ with symbol $\Phi(z)=\frac{(1-z)^{\alpha}}{z}$.

Proof. Given $u \in \ell^{2}\left(\mathbb{N}_{0}\right)$, we have by [22, Proposition $\left.2.9(\mathrm{v})\right]$ that $\Delta^{\alpha} u=\Delta\left(k^{1-\alpha} * u\right)=\Delta k^{1-\alpha} *$ $u+\tau_{1} u$ where $\tau_{1}$ denotes the translation operator. Using Young's convolution inequality, we get

$$
\left\|\Delta^{\alpha} u\right\|_{2} \leq\left\|\Delta k^{1-\alpha} * u\right\|_{2}+\|u\|_{2} \leq\left\|\Delta k^{1-\alpha}\right\|_{1}\|u\|_{2}+\|u\|_{2}
$$

where we have used [22, Proposition 3.1 (viii)] and the estimate

$$
\Delta k^{1-\alpha}(n) \sim \frac{C}{n^{\alpha+1}} .
$$

which follows from [22, Lemma $3.2(\mathrm{i})]$. This proves that the operator $\Delta^{\alpha}$ is bounded on $\ell^{2}\left(\mathbb{N}_{0}\right)$.

In view of 77 we have $\Phi(z)=\frac{1}{z}+\sum_{j=0}^{\infty} \frac{(-\alpha) k^{1-\alpha}(j) z^{j}}{(j+1)}$. Let denote

$$
\varphi(z)=\sum_{j=0}^{\infty} \frac{(-\alpha) k^{1-\alpha}(j) z^{j}}{(j+1)}
$$


then we get

$$
z \varphi(z)=\sum_{j=0}^{\infty} \frac{(-\alpha) k^{1-\alpha}(j) z^{j+1}}{(j+1)}
$$

and then using (4) we have

$$
[z \varphi(z)]^{\prime}=-\alpha \sum_{j=0}^{\infty} k^{1-\alpha}(j) z^{j}=-\alpha(1-z)^{\alpha-1}
$$

After integrating once, we obtain

$$
z \varphi(z)=(1-z)^{\alpha}+c
$$

Replacing $z=0$ in (8) we get $c=-1$. As a consequence $\varphi(z)=\frac{(1-z)^{\alpha}}{z}-\frac{1}{z}$. Finally, it follows that $\Phi(z)=\frac{1}{z}+\varphi(z)=\frac{(1-z)^{\alpha}}{z}$ is the symbol of the Toeplitz operator.

In order to prove chaos of the operator $\Delta^{\alpha}$ for $0<\alpha \leq 1$ we use Theorem 4.2. Let first check condition $(i)$, that is, $\Phi(z)=\frac{(1-z)^{\alpha}}{z}$ is univalent in $\mathbb{D} \backslash\{0\}$. Indeed, using Theorem 2.4 it suffices to show that

$$
\Re\left(\frac{D^{1} \Phi(z)}{\Phi(z)}-2\right)<0, \text { for }|z|<1,
$$

where $D^{n} \Phi(z)=\frac{1}{z}\left(z^{n+1} \frac{\Phi(z)}{n !}\right)^{(n)}, n \in \mathbb{N}_{0}$. An easy computation shows that $D^{1} \Phi(z)=\frac{(1-z)^{\alpha}}{z}-\alpha(1-$ $z)^{\alpha-1}$ and taking $z=a+i b$ with $-1<a<1$ it follows that

$$
\Re\left(\frac{D^{1} \Phi(z)}{\Phi(z)}-2\right)=\Re\left(-1-\frac{\alpha z}{1-z}\right)=\frac{-(1-a)^{2}+(\alpha-1) b^{2}-\alpha a(1-a)}{(1-a)^{2}+b^{2}} .
$$

It is clear that $\Re\left(\frac{D^{1} \Phi(z)}{\Phi(z)}-2\right)<0$ if and only if $-(1-a)^{2}+(\alpha-1) b^{2}-\alpha a(1-a)<0$ and this last assertion holds since $0<\alpha<1$ and $-1<a<1$. It only remains to show condition (ii) in Theorem 4.2 Actually, we will show that $\left.I:=\left[-2^{\alpha}, 0\right] \subset \mathbb{C} \backslash \Phi(\mathbb{D})\right)$. Indeed, if $z \in \mathbb{D}$ is so that $\Phi(z)=z^{\prime} \in I$, then $\arg \left(z^{\prime}\right)=\pi$. This implies $\arg \left((1-z)^{\alpha}\right)=\theta+\pi$, where $\theta=\arg (z)$, a contradiction.

Remark 5.2. We observe that the symbol of the Toeplitz operator $\Delta^{\alpha}$ coincides with the symbol of the explicit Euler approximation scheme for the Riemann-Liouville fractional differential operator [25].

A very interesting consequence of this theorem when combined with the transference principle stated in section 3 is the following corollary.

Corollary 5.3. For any $0<\alpha \leq 1$ and $a>0$, the nabla difference operator $\nabla_{a}^{\alpha}$ is chaotic in $\ell^{2}\left(\mathbb{N}_{a}\right)$.

Proof. It is a direct consequence of Theorem 3.5 and [24, Proposition 2.24].

Let $b \in \ell^{1}\left(\mathbb{N}_{0}\right)$ be a summable sequence and define its Gelfand transform by

$$
\delta(z):=\sum_{n=0}^{\infty} b(n) z^{n}, \quad z \in \mathbb{D} .
$$

In what follows, we will always assume that the radial $\operatorname{limit} \delta(\xi):=\lim _{z \rightarrow \xi} \delta(z)$ exists for all $\xi \in$ $\mathbb{T} \backslash\{ \pm 1\}$.

The next result in an important criterion for chaos of a specific operator that covers many nonlocal difference operators as we will see later.

Theorem 5.4. Let $b \in \ell^{1}\left(\mathbb{N}_{0}\right)$ be given and $T_{b}: \ell^{2}\left(\mathbb{N}_{0}\right) \rightarrow \ell^{2}\left(\mathbb{N}_{0}\right)$ given by

$$
T_{b} u(n)=\sum_{j=0}^{\infty} b(j) B^{j} u(n), \quad n \in \mathbb{N}_{0},
$$


where $B$ denotes the backward shift operator. Then $T_{b}$ defines a bounded operator on $\ell^{2}\left(\mathbb{N}_{0}\right)$ and the following assertions are equivalent

(i) $T_{b}$ is chaotic;

(ii) $\delta(\mathbb{D}) \cap \mathbb{T} \neq \emptyset$.

Proof. We observe that $\varphi_{b}(B)=T_{b}$ where

$$
\varphi_{b}(z):=\sum_{j=0}^{\infty} b(j) z^{j}=\delta(z),
$$

defines an holomorphic function on the disk $\mathbb{D}$, because $b \in \ell^{1}\left(\mathbb{N}_{0}\right)$. Moreover, we have that $\varphi(B)$ defines a bounded operator on $\ell^{2}\left(\mathbb{N}_{0}\right)$ and, by Theorem 2.3 . we deduce that $T_{b}$ is chaotic if and only if $\delta(\mathbb{D}) \cap \mathbb{T} \neq \emptyset$. This proves the theorem.

In what follows we study chaos for relevant nonlocal difference operators arising in the study of time-stepping schemes for fractional operators 25. They are defined by a convolution operator $\partial_{b}^{\alpha}: \ell^{2}\left(\mathbb{N}_{0}\right) \rightarrow \ell^{2}\left(\mathbb{N}_{0}\right)$ given by

$$
\partial_{b}^{\alpha} u(n):=(b * u)(n), \quad n \in \mathbb{N}_{0},
$$

where $b \in \ell^{1}\left(\mathbb{N}_{0}\right)$ is a real valued sequence, implicitly defined by the generating series

$$
\delta(\xi):=\sum_{n=0}^{\infty} b(n) \xi^{n}, \quad \xi \in \mathbb{T},
$$

and where $\delta(\xi)$ represents the symbol of the scheme. An easy calculation shows that the dual operator of $\partial_{b}^{\alpha}$ in $\ell^{2}\left(\mathbb{N}_{0}\right)$, is given by

$$
\left(\partial_{b}^{\alpha}\right)^{*} u(n)=T_{b} u(n)=\sum_{j=0}^{\infty} b(j) B^{j} u(n), \quad n \in \mathbb{N}_{0} .
$$

Surprisingly, in some cases, the above operator is known. For instance, consider $b(n)=k^{-\alpha}(n)$. Then it perfectly coincides with the Weil fractional difference operator of order $\alpha>0$, denoted by $W^{\alpha}$. See [1, Definition 3.1 and Theorem 3.2].

As a first example, we consider $\delta(\xi)=1-\xi$ that represents the Backward Euler scheme [25]. A simple inspection comparing with the series (11) shows that $b(n)=e_{0}(n)-e_{1}(n)$. Therefore

$$
\partial_{b}^{\alpha} u(n)=u(n)-u(n-1), \quad n=1,2, \ldots
$$

and $\partial_{b}^{\alpha} u(0)=u(0)$. The associated matrix in the canonical basis, takes the form

$$
\partial_{b}^{\alpha} e_{l}(n)=\left\{\begin{array}{cl}
1 & \text { if } n=l \\
-1 & \text { if } n=l+1 \\
0 & \text { otherwise }
\end{array}\right.
$$

where $n, l \in \mathbb{N}_{0}$. We can easily verify that for $b(n)$ given as before, we obtain

$$
\left(\partial_{b}^{\alpha}\right)^{*} u(n)=u(n)-u(n+1)=-\Delta u(n), \quad n \in \mathbb{N}_{0} .
$$

Consequently, the associated matrix to the dual operator is represented, as predicted, by

$$
\left(\partial_{b}^{\alpha}\right)^{*} e_{l}(n)=\left\{\begin{array}{cl}
1 & \text { if } n=l \\
-1 & \text { if } n=l-1 \\
0 & \text { otherwise. }
\end{array}\right.
$$

We observe that such operator is chaotic [24, Theorem 4.43]. For a given $\alpha>0$ we consider now the fractional backward Euler scheme with symbol $\delta(\xi)=\tau^{-\alpha}(1-\xi)^{\alpha}$ where $\tau>0$ denotes the step size 
of the scheme [25]. In such case, we obtain the sequence kernel $b_{\tau}(n)=\tau^{-\alpha} k^{-\alpha}(n)$, and then we can consider the following nonlocal operator

$$
\partial_{k}^{\alpha} u(n)=\sum_{j=0}^{n} \tau^{-\alpha} k^{-\alpha}(n-j) u(j) .
$$

It is remarkable that $\left(\partial_{k}^{\alpha}\right)^{*}=W_{\tau}^{\alpha}$ corresponds to the Weil fractional difference operator or order $\alpha>0$ recently studied in [1] (with step size $\tau=1$ ) and whose matrix is represented by

$$
W_{\tau}^{\alpha} e_{l}(n)= \begin{cases}\tau^{-\alpha} k^{-\alpha}(n-l) & \text { if } n \geq l \\ 0 & \text { otherwise. }\end{cases}
$$

We prove the following interesting result that shows that chaos for $W_{\tau}^{\alpha}$ depends on the step size $\tau$ but not on the fractional order derivative, in contrast with the nonlocal operator $\Delta^{\alpha}$.

Theorem 5.5. For any $\alpha>0$ the Weil difference operator $W_{\tau}^{\alpha}$ is chaotic on $\ell^{2}\left(\mathbb{N}_{0}\right)$ if and only if $0<\tau<2$.

Proof. According to Theorem 5.4 it is enough to prove that $\delta_{\tau}(\mathbb{D}) \cap \mathbb{T} \neq \emptyset$ if and only if $0<\tau<2$ where $\delta_{\tau}(z)=\tau^{-\alpha}(1-z)^{\alpha}$. Indeed, we note that $w \in \delta_{\tau}(\mathbb{D}) \cap \mathbb{T}$ if and only if $w=\tau^{-\alpha}(1-z)^{\alpha}$ where $|w|=1$ and $|z|<1$. Then, the identity $\left|1-\tau w^{1 / \alpha}\right|=|z|$ shows that the complex number $\tau w^{1 / \alpha}$ must belong to the disk of center 1 and radius 1 . Consequently, $0<\tau<2$ iff $\delta_{\tau}(\mathbb{D}) \cap \mathbb{T} \neq \emptyset$.

In what follows, we consider the fractional second order backward difference scheme given by the symbol

$$
\delta(\xi)=\tau^{-\alpha}\left(\frac{3}{2}-2 \xi+\frac{1}{2} \xi^{2}\right)^{\alpha}=\tau^{-\alpha}\left(\frac{3}{2}\right)^{\alpha}(1-z)^{\alpha}\left(1-\frac{z}{3}\right)^{\alpha} .
$$

It was recently proved in 29 that for such time stepping scheme, the sequence $b(n)$ is given by

$$
b(n)=\frac{3}{2} e_{0}(n)-2 e_{1}(n)+\frac{1}{2} e_{2}(n),
$$

in case $\alpha=1$ and

$$
b(n)=\left(\frac{3}{2}\right)^{\alpha} \sum_{j=0}^{n} k^{-\alpha}(n-j) \frac{1}{3^{j}} k^{-\alpha}(j),
$$

in case $\alpha \neq 1$. We check that $b \in \ell^{1}\left(\mathbb{N}_{0}\right)$. Indeed, define $c(n):=\frac{k^{-\alpha}(n)}{3^{n}}$. Then we have $c(n) \sim \frac{C}{n^{1+\alpha} 3^{n}}$ because $k^{-\alpha}(n) \sim \frac{C}{n^{1+\alpha}}$. Therefore, both sequences $c$ and $k^{-\alpha}$ belong to the Lebesgue space $\ell^{1}\left(\mathbb{N}_{0}\right)$. This facts, together with Young's inequality for the convolution, imply the assertion.

For such sequence $b(n)$, we consider the following scheme

$$
\partial_{b}^{\alpha} u(n)=\tau^{-\alpha}(b * u)(n)
$$

where $\tau>0$ is the step size. 1.

The next result shows, once again, the independence of chaos from the fractional order. See Figure

Theorem 5.6. The operator $T_{b}$, which is the dual of the operator that defines the fractional second order backward difference scheme with step size $\tau$, is chaotic on $\ell^{2}\left(\mathbb{N}_{0}\right)$ if and only if $0<\tau<4$.

Proof. According to Theorem 5.4 it is enough to prove that $\delta(\mathbb{D}) \cap \mathbb{T} \neq \emptyset$ if and only $0<\tau<4$, where $\delta(z)$ is given by the symbol $(15)$, with $z \in \mathbb{D}$. By the maximum principle, we have $\sup _{z \in \mathbb{D}}|\delta(z)|=$ $\max _{z \in \mathbb{T}}|\delta(z)|$ which, by the identity $(15)$, is attained at $z=-1$. Therefore, $\sup _{z \in \mathbb{D}}|\delta(z)|=4^{\alpha} \tau^{-\alpha}$. It shows that the set $\delta(\mathbb{D})$ intersects the unit circle if and only if $4^{\alpha} \tau^{-\alpha}>1$ which proves the assertion of the theorem. 


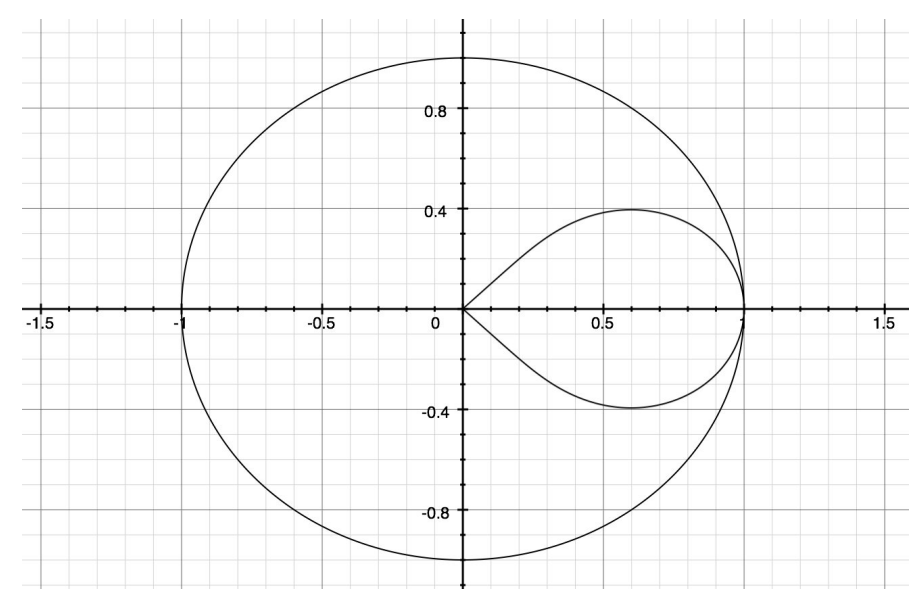

Figure 1. $\delta(\mathbb{D})$ for the symbol of the fractional second order backward difference scheme with $\alpha=\frac{1}{2}$ and $\tau=4$, and $\mathbb{T}$.

Finally, we consider the fractional Crank- Nicholson stepping scheme, whose symbol is given by

$$
\delta(\xi)=\tau^{-\alpha} \frac{(1-\xi)^{\alpha}}{1-\frac{\alpha}{2}+\frac{\alpha}{2} \xi},
$$

where $0<\alpha<2$. By [29, Example 4.4] we have that the sequence $b(n)$ is given by

$$
b(n)=\tau^{-\alpha} \frac{2}{2-\alpha} \sum_{j=0}^{n} k^{-\alpha}(n-j)\left(\frac{\alpha}{\alpha-2}\right)^{j} .
$$

For the range $0<\alpha<1$ we define the sequence $c(n):=\left(\frac{\alpha}{\alpha-2}\right)^{n}$. Since $\alpha<1$ we have $c \in \ell^{1}\left(\mathbb{N}_{0}\right)$ and since

$$
k^{-\alpha}(n) \sim \frac{C}{n^{\alpha+1}} .
$$

which follows from [22, Lemma 3.2 (viii)] we deduce $k^{-\alpha} \in \ell^{1}\left(\mathbb{N}_{0}\right)$. Therefore, Young's inequality for the convolution implies that $b \in \ell^{1}\left(\mathbb{N}_{0}\right)$.

In the case $0<\alpha<1$ we arrive at the following result, which reveals a surprising connection of chaos not only with the step size but also with the fractional order $\alpha$.

Theorem 5.7. Let $0<\alpha<1$. The operator $T_{b}$, which is the dual of the operator that defines the fractional Crank-Nicholson scheme with step size $\tau$, is chaotic on $\ell^{2}\left(\mathbb{N}_{0}\right)$ if and only if $0<\tau<$ $\frac{2}{(1-\alpha)^{1 / \alpha}}$.

Proof. By Theorem 5.4 we have to prove that the property $\delta(\mathbb{D}) \cap \mathbb{T} \neq \emptyset$ is valid for the holomorphic function $\delta(z)=\tau^{-\alpha} \frac{(1-z)^{\alpha}}{1-\frac{\alpha}{2}+\frac{\alpha}{2} z}$ defined by the Crank-Nicholson scheme. Observe that $\delta(z)$ is a conformal map satisfying $\delta(1)=0$ and $\delta(-1)=\tau^{-\alpha} \frac{2^{\alpha}}{1-\alpha}$. Moreover, by the maximum principle, we have that $\sup _{z \in \mathbb{D}}|\delta(z)|=\max _{z \in \mathbb{T}}|\delta(z)|$. Therefore, the maximum value of the set $\delta(\mathbb{D})$ is attained at $\delta(-1)$. Hence, we have non empty intersection with the unit circle if and only if $\delta(-1)>1$ which means $0<\tau<\frac{2}{(1-\alpha)^{1 / \alpha}}$.

\section{Data Availability}

Data sharing not applicable no new data generated.

\section{Acknowledgments}


C. Lizama is partially supported by FONDECYT grant number 1180041 and DICYT, Universidad de Santiago de Chile, USACH.

M. Murillo-Arcila is supported by MICINN and FEDER, Projects MTM2016-75963-P and PID2019105011GB-I00, and by Generalitat Valenciana, Project GVA/2018/110.

A. Peris is supported by MICINN and FEDER, Projects MTM2016-75963-P and PID2019-105011GBI00, and by General- itat Valenciana, Project PROMETEO/2017/102.

\section{REFERENCES}

[1] L. Abadias and P.J. Miana. Generalized Cesáro operators, fractional finite differences and Gamma functions. J. Funct. Anal., 274 (5)(2018), 1424-1465.

[2] F. M. Atici and P. W. Eloe. A transform method in discrete fractional calculus. Int. J. Difference Equ. 2 (2) (2007), $165-176$.

[3] F. M. Atici and P. W. Eloe. Discrete fractional calculus with the nabla operator. Electron. J. Qual. Theory Differ. Equ., 3 (2009), 1-12.

[4] F. M. Atici and P. W. Eloe Two-point boundary value problems for finite fractional difference equations, J. Difference Equ. Appl. 17 (2011), 445-456.

[5] F. M. Atici and P. W. Eloe, Initial value problems in discrete fractional calculus. Proc. Amer. Math. Soc., 137(3) (2009), 981-989.

[6] F. M. Atici and S. Sengul, Modeling with fractional difference equations. J. Math. Anal. Appl., 369 (2010),1-9.

[7] J. Banks, J. Brooks, G. Cairns, G. Davis and P. Stacey, On Devaney's definition of chaos. Amer. Math. Monthly, 99(4) (1992),332-334.

[8] A. Baranov and A. Lishanskii. Hypercyclic Toeplitz Operators. Results. Math. 70 (2016), 337-347.

[9] F. Bayart and É. Matheron, Dynamics of linear operators, Cambridge University Press, Cambridge, 2009.

[10] R. deLaubenfels and H. Emamirad, Chaos for functions of discrete and continuous weighted shift operators, Ergodic Theory Dynam. Systems 21 (2001), 1411-1427.

[11] R. L. Devaney. An introduction to chaotic dynamical systems, Benjamin/Cummings, Menlo Park, CA, 1986; second edition, Addison-Wesley, Redwood City, CA, 1989.

[12] M. Edelman. Caputo standard $\alpha$-family of maps: Fractional difference vs. fractional. Chaos 24, 023137 (2014); https://doi.org/10.1063/1.4885536.

[13] M. Edelman. On the fractional Eulerian numbers and equivalence of maps with long term power-law memory (integral Volterra equations of the second kind) to Grnwald-Letnikov fractional difference (differential) equations. Chaos 25, 073103 (2015); doi: 10.1063/1.4922834.

[14] M. Edelman. Fractional maps and fractional attractors. Part II: Fractional difference Caputo $\alpha$-families of maps. Discontinuity, Nonlinearity, and Complexity 4(4) (2015) 391-402.

[15] L. Erbe, C. S. Goodrich, B. Jia and A. C. Peterson. Survey of the qualitative properties of fractional difference operators: monotonicity, convexity, and asymptotic behavior of solutions. Adv. Difference Equ., 2016 (43), (2016), doi: 10.1186/s13662-016-0760-3.

[16] R. A. C. Ferreira. A discrete fractional Gronwall inequality. Proc. Amer. Math. Soc. 140 (2012), 1605-1612.

[17] R. A. C. Ferreira. Existence and uniqueness of solution to some discrete fractional boundary value problems of order less than one. J. Difference Equ. Appl. 19 (2013), 712-718.

[18] M. R. Ganigi and B. A. Uralegaddi. New criteria for meromorphic univalent functions, Bull. Math. dela Soc. Sci. Math de la R.S. de Roumanie, 33(81) (1989), 9-13.

[19] C. S. Goodrich and A. C. Peterson. Discrete Fractional Calculus, Springer International Publishing, 2015, doi: 10.1007/978-3-319-25562-0.

[20] C. S. Goodrich. On discrete sequential fractional boundary value problems. J. Math. Anal. Appl. 385 (2012), $111-124$.

[21] C. S. Goodrich. A convexity result for fractional differences. Appl. Math. Lett. 35 (2014), 58-62.

[22] C. Goodrich and C. Lizama. A transference principle for nonlocal operators using a convolutional approach: Fractional monotonicity and convexity. Israel J. Math., 236 (2020), 533-589.

[23] H. L. Gray and N.F. Zhang. On a new definition of the fractional difference. Math. of Comput., 50 (182) (1988), 513-529.

[24] K.G. Grosse-Erdmann and A. Peris Linear Chaos. Universitext, Springer-Verlag London Ltd., London, 2011.

[25] B. Jin, B. Li and Z. Zhou. Discrete maximal regularity of time-stepping schemes for fractional evolution equations. Numer. Math. 138(1) (2018), 101-131.

[26] L. Kexue. J. Peng and J. Junxiong, Cauchy problems for fractional differential equations with Riemann Liouville fractional derivatives. J. Functional Analysis, 263(2) (2012), 476-510.

[27] C. Lizama. The Poisson distribution, abstract fractional difference equations, and stability. Proc. Amer. Math. Soc. 145(9) (2017), 3809-3827.

[28] C. Lizama. $\ell_{p}$-maximal regularity for fractional difference equations on UMD spaces. Math. Nach., 288(17/18) (2015), 2079-2092. 
[29] C. Lizama and M. Murillo. On a connection between discrete maximal regularity for Volterra equations and nonlocal time-stepping schemes. Discrete Cont. Dyn. Syst. Series A, 40(1) (2020), 509-528.

[30] R. A. Martínez-Avendaño and O. Zatarain-Vera, Subspace hypercyclicity for Toeplitz operators. J. Math. Anal. Appl., 422(1) (2015), 772-775.

[31] F. Martínez-Giménez, Chaos for power series of backward shift operators. Proc. Amer. Math. Soc., 135(6) (2007), $1741-1752$.

[32] A. G. Radwan, S. H. AbdElHaleem and S. K. Abd-El-Hafiz. Symmetric encryption algorithms using chaotic and non-chaotic generators: A review. J. Adv. Research 7 (2016), 193-208.

[33] A.G. Radwan, K. Moaddy, K.N. Salama, S. Momani and I. Hashim. Control and switching synchronization of fractional order chaotic systems using active control technique. J. Adv. Research 5 (2014), 125-132.

[34] G.-C. Wu and D. Baleanu, Discrete fractional logistic map and its chaos. Nonlinear Dynamics, 75(1-2) (2014), $283-287$.

[35] G.-C. Wu, D. Baleanu and S. D. Zeng, Discrete chaos in fractional sine and standard maps. Physics Letters A, 378 (5-6) (2014), 484-487.

[36] A. Zygmund. Trigonometric series. 2nd ed. Vol. I, II. Cambridge University Press, New York, 1959. 